CARPATHIAN J. MATH.

Volume 37 (2021), No. 2,

Pages 185 - 194
Online version at https : //www . carpathian. cunbm . utcluj. ro/

Print Edition: ISSN 1584 - 2851; Online Edition: ISSN 1843 - 4401

DOI: https://doi.org/10.37193/CJM.2021.02.04

Dedicated to Prof. Ioan A. Rus on the occasion of his $85^{\text {th }}$ anniversary

\title{
Approximation of random functions by stochastic Bernstein polynomials in capacity spaces
}

\author{
SORIN G. GAL and CONSTANTIN P. NiCUlESCU
}

\begin{abstract}
Given a submodular capacity space, we firstly obtain a quantitative estimate for the uniform convergence in the Choquet $p$-mean, $1 \leq p<\infty$, of the multivariate stochastic Bernstein polynomials associated to a random function. Also, quantitative estimates concerning the uniform convergence in capacity in the univariate case are given.
\end{abstract}

\section{INTRODUCTION}

In this paper we extend some old and new results on the approximation of random functions by Bernstein random polynomials to the framework of capacities and Choquet's theory of integrability. As is well known, these polynomials are among the most studied and the most interesting polynomials used in the probabilistic framework of approximation theory. We mention here the classical book of Lorentz [21] and the papers of Onicescu and Istrățescu [22], [23], Cenuşă and Săcuiu [3], Gal [7], [8], and Gal and Villena [17]. In the very recent papers of Adell and Cárdenas-Morales [2], Sun and $\mathrm{Wu}$ [25], $\mathrm{Wu}$, Sun and $\mathrm{Ma}$ [26] and $\mathrm{Wu}$ and Zhou [27], quantitative estimates for approximation in probability of deterministic functions by random Bernstein polynomials were obtained.

The papers cited above have motivated us to study the extension of the approximation properties of random Bernstein polynomials in the much more general framework provided by capacity spaces and the Choquet integral. Unlike the case of probability measures, the capacities are nonadditive set functions, and precisely the lack of additivity makes them useful in risk theory (especially in decision making under risk and uncertainty). See Föllmer and Schied [6] and Grabisch [18].

It is worth mentioning that the present paper belongs to the recent direction of study concerning to what extent various properties in Real and Functional Analysis can be extended by replacing the probability measure with a nonadditive set function (capacity) and the linear Lebesgue integral with the nonlinear Choquet integral. See, e.g., the very recent papers Gal [9], Gal and Opris [15], Gal and Trifa [16], Gal and Iancu [10], Gal and Niculescu [11]-[14].

In Section 2 we present preliminaries on capacities and Choquet integral. Section 3 is devoted to a description of various concepts of continuity and convergence of random functions in the setting of capacities and Choquet integral. Section 4 deals with approximation results by stochastic Bernstein polynomials of several variables in the framework of the Choquet integral. Our main result is Theorem 4.2, devoted to the approximation in the Choquet $p$-mean, $p \in[1, \infty)$. In the probabilistic case (and for functions of one real

Received: 24.10.2020. In revised form: 24.03.2021. Accepted: 31.03.2021

2010 Mathematics Subject Classification. 60G99, 41A10, 41A36, 28 A25.

Key words and phrases. Choquet integral, submodular capacity, random Bernstein polynomials, approximation in Choquet p-mean, approximation in capacity, Choquet $L^{p}$-modulus of continuity.

Corresponding author: Sorin G. Gal; galso@uoradea.ro 
variable and $p=1$ for Theorem 4.2), these results were previously proved respectively in Cenuşă and Săcuiu [3] and Onicescu and Istrăţescu [22]. In section 5 we obtain quantitative estimates for the approximation in capacity by univariate Bernstein-type random polynomials, generalizing recent results due to Adell and Cárdenas-Morales [2], Sun and $\mathrm{Wu}$ [25], Wu, Sun and Ma [26] and $\mathrm{Wu}$ and Zhou [27], who considered only the framework of probability measures and of deterministic functions.

\section{Preliminaries ON CAPACITIES AND CHOQUet INTEGRAL}

For the convenience of the reader we will briefly recall some basic facts concerning Choquet's theory of integrability with respect to a nondecreasing set function (not necessarily additive). Full details are to be found in the books of Denneberg [5] and Grabisch [18].

Let $(\Omega, \mathcal{A})$ be an arbitrarily fixed measurable space, that is, a nonempty abstract set $\Omega$ endowed with a $\sigma$-algebra $\mathcal{A}$ of subsets of $\Omega$.

Definition 2.1. A set function $\mu: \mathcal{A} \rightarrow \mathbb{R}_{+}$is called a capacity if it verifies the following two conditions:

(a) $\mu(\emptyset)=0$ and $\mu(\Omega)=1$;

(b) $\mu(A) \leq \mu(B)$ for all $A, B \in \mathcal{A}$, with $A \subset B$.

A capacity $\mu$ is called subadditive if

$$
\mu(A \bigcup B) \leq \mu(A)+\mu(B)
$$

and submodular (or strongly subadditive) if

$$
\mu(A \bigcup B)+\mu(A \bigcap B) \leq \mu(A)+\mu(B)
$$

for all $A, B \in \mathcal{A}$.

A simple way to construct nontrivial examples of submodular capacities is to start with a probability measure $P: \mathcal{A} \rightarrow[0,1]$ and to consider any nondecreasing concave function $u:[0,1] \rightarrow[0,1]$ such that $u(0)=0$ and $u(1)=1$; for example, one may chose $u(t)=t^{a}$ with $0<\alpha<1$.Then $\mu=u(P)$ is a submodular capacity on the $\sigma$-algebra $\mathcal{A}$, called a distorted probability.

The capacity spaces (that is, the triplets $(\Omega, \mathcal{A}, \mu)$, where $\Omega$ is a nonempty abstract set endowed with a $\sigma$-algebra $\mathcal{A}$ of subsets of $\Omega$ and $\mu: \mathcal{A} \rightarrow \mathbb{R}_{+}$is a capacity) represent a generalization of the classical concept of probability space.

To a capacity space $(\Omega, \mathcal{A}, \mu)$ one can attach several spaces of functions, starting with the space $L^{0}(\Omega, \mathcal{A}, \mu)$ of all random variables $f: \Omega \rightarrow \mathbb{R}$ (that is, of all functions $f$ verifying the condition of $\mathcal{A}$-measurability, $f^{-1}(A) \in \mathcal{A}$ for every Borel subset $A \subset \mathbb{R}$ ) and continuing with the analogs of the classical Lebesgue spaces $L^{p}(\Omega, \mathcal{A}, \mu)$ (for $\left.1 \leq p<\infty\right)$, when the capacity $\mu$ is submodular.

The key ingredient is the integrability of random variables $f \in L^{0}(\Omega, \mathcal{A}, \mu)$ with respect to the capacity $\mu$.

Definition 2.2. The Choquet integral of a random variable $f: \Omega \rightarrow \mathbb{R}$ on a set $A \in \mathcal{A}$ is defined by the formula

$$
\text { (C) } \begin{aligned}
\int_{A} f \mathrm{~d} \mu & =\int_{0}^{+\infty} \mu(\{\omega \in \Omega: f(\omega)>t\} \cap A) \mathrm{d} t \\
& +\int_{-\infty}^{0}[\mu(\{\omega \in \Omega: f(\omega)>t\} \cap A)-\mu(A)] \mathrm{d} t,
\end{aligned}
$$

where the integrals in the right hand side are generalized Riemann integrals. 
If $(C) \int_{A} f \mathrm{~d} \mu$ exists in $\mathbb{R}$, then $f$ is called Choquet integrable on $A$.

The Choquet integral agrees with the Lebesgue integral in the case of probabilistic measures, see Denneberg [5], p. 62.

The next remark summarizes basic properties of the Choquet integral used in the next sections.

Remark 2.1. (a) If $f, g \in L^{0}(\Omega, \mathcal{A}, \mu)$ are Choquet integrable on $A$, then

$$
\begin{gathered}
f \leq g \text { implies (C) } \int_{A} f \mathrm{~d} \mu \leq(\mathrm{C}) \int_{A} g \mathrm{~d} \mu \quad \text { (monotonicity) } \\
\text { (C) } \int_{A} a f \mathrm{~d} \mu=a \cdot(\mathrm{C}) \int_{A} f \mathrm{~d} \mu \text { for all } a \geq 0 \quad \text { (positive homogeneity). }
\end{gathered}
$$

(b) If $\mu$ is a submodular capacity, then the associated Choquet integral is subadditive, that is,

$$
\text { (C) } \int_{A}(f+g) \mathrm{d} \mu \leq(\mathrm{C}) \int_{A} f \mathrm{~d} \mu+(\mathrm{C}) \int_{A} g \mathrm{~d} \mu
$$

for all functions $f$ and $g$ integrable on $A$, see [5], Theorem 6.3, p. 75 .

The analogues of the Lebesgue spaces in the context of capacities can be introduced for $1 \leq p<+\infty$ via the formulas

$$
\mathcal{L}^{p}(\Omega, \mathcal{A}, \mu)=\left\{f: f \in L^{0}(\Omega, \mathcal{A}, \mu) \text { and }(C) \int_{\Omega}|f(\omega)|^{p} \mathrm{~d} \mu<+\infty\right\} .
$$

When $\mu$ is a submodular capacity, the quotient space $L^{p}(\Omega, \mathcal{A}, \mu)=\mathcal{L}^{p}(\Omega, \mathcal{A}, \mu) / \mathcal{N}_{p}$, where $\mathcal{N}_{p}=\left\{f \in \mathcal{L}^{p}(\Omega, \mathcal{A}, \mu):\left((C) \int_{\Omega}|f(\omega)|^{p} \mathrm{~d} \mu\right)^{1 / p}=0\right\}$, becomes a normed vector space relative to the norm $\|f\|_{L^{p}(\Omega, \mathcal{A}, \mu)}=\left((C) \int_{\Omega}|f(\omega)|^{p} \mathrm{~d} \mu\right)^{1 / p}$, see [5], Proposition 9.4, p. 109, for $p=1$ and ibidem $\mathrm{p}$. 115 for arbitrary $p \geq 1$.

\section{CONTINUITY OF RANDOM FUNCTIONS ASSOCIATED TO A CAPACITY SPACE}

Given a capacity space $(\Omega, \mathcal{A}, \mu)$ and a subset $D$ of the Euclidean space $\mathbb{R}^{N}$, we will refer to the functions $F: D \rightarrow L^{0}(\Omega, \mathcal{A}, \mu)$ as random functions. It is also usual to interpret $F$ as a stochastic/random process $F: D \times \Omega \rightarrow \mathbb{R}, F(\mathbf{x}, \omega)=F(\mathbf{x})(\omega)$. For fixed $\omega, F(\mathbf{x}, \omega)$ is a deterministic function of $\mathbf{x}$, called a sample function.

Following the case of probabilistic spaces one can consider several kinds of continuity and of approximation, of interest for us being the following ones.

Definition 3.3. A random function $F$ is continuous in capacity at the point $\mathbf{x}_{0} \in D$, if $\mathbf{x} \rightarrow \mathbf{x}_{0}$ implies $F(\mathbf{x}) \rightarrow F\left(\mathbf{x}_{0}\right)$ in capacity, that is, for every $\varepsilon>0$ and $\eta>0$ there exists $\delta=\delta\left(\varepsilon, \eta, \mathbf{x}_{0}\right)>0$ such that

$$
\mu\left(\left\{\omega \in \Omega:\left|F(\mathbf{x}, \omega)-F\left(\mathbf{x}_{0}, \omega\right)\right| \geq \varepsilon\right\}\right)<\eta
$$

whenever $\mathbf{x} \in D$ and $\left\|\mathbf{x}-\mathbf{x}_{0}\right\|<\delta$.

If $\delta$ depends only on $\varepsilon$ and $\eta$ and (3.2) holds for all $x$ and $x_{0}$, then the random function $F$ is called uniformly continuous in capacity.

A random function $F: D \rightarrow L^{p}(\Omega, \mathcal{A}, \mu), 1 \leq p<+\infty$, is called continuous in the Choquet-mean of order $p$ (shortly Choquet $p$-mean) at the point $\mathbf{x}_{0} \in D$, if for every $\varepsilon>0$ there exists $\delta=\delta\left(\varepsilon, \mathbf{x}_{0}\right)>0$, such that for all $\mathbf{x} \in D$ with $\left\|\mathbf{x}-\mathbf{x}_{0}\right\|<\delta$, we have

$$
\text { (C) } \int_{\Omega}\left|F(\mathbf{x}, \omega)-F\left(\mathbf{x}_{0}, \omega\right)\right|^{p} \mathrm{~d} \mu<\varepsilon .
$$

If $\delta$ depends only on $\varepsilon$ and (3.3) holds for all $x$ and $x_{0}$, then the random function $F$ is called uniformly continuous in Choquet $p$-mean. 
A sequence $\left(F_{n}\right)_{n}$ of random functions converges in capacity to the random function $F$ at $\mathbf{x} \in D$, if for every $\varepsilon, \eta>0$, there exists $N(\varepsilon, \eta, \mathbf{x}) \in \mathbb{N}$ such that for all $n \geq N(\varepsilon, \eta, \mathbf{x})$ we have

$$
\mu\left(\left\{\omega \in \Omega:\left|F_{n}(\mathbf{x}, \omega)-F(\mathbf{x}, \omega)\right| \geq \varepsilon\right\}\right)<\eta .
$$

If $N(\varepsilon, \eta, \mathbf{x})$ does not depend on $\mathbf{x}$, then we say that $\left(F_{n}\right)_{n}$ converges uniformly in capacity to $F$.

A sequence $\left(F_{n}\right)_{n}$ of random functions converges in Choquet $p$-mean to the random function $F$, if for every $\varepsilon>0$ and $\mathbf{x} \in D$, there exists $N(\varepsilon, \mathbf{x}) \in \mathbb{N}$ such that for all $n \geq N(\varepsilon, \mathbf{x})$ we have

$$
\text { (C) } \int_{\Omega}\left|F_{n}(\mathbf{x}, \omega)-F(\mathbf{x}, \omega)\right|^{p} \mathrm{~d} \mu<\varepsilon .
$$

If $N(\varepsilon, \mathbf{x})$ does not depend on $\mathbf{x}$, then we will say that $\left(F_{n}\right)_{n}$ converges uniformly to $F$ in the Choquet $p$-mean.

For $1 \leq p<+\infty$ and $\delta_{i} \geq 0, i=1, \ldots, N$, the multivariate Choquet $L^{p}$-modulus of continuity of $F$ will be defined by

$\Gamma\left(f: \delta_{1}, \ldots, \delta_{N}\right)_{p}=\left(\sup _{\left|t_{i}-s_{i}\right| \leq \delta_{i}, i=1, \ldots N}(C) \int_{\Omega}\left|F\left(t_{1}, \ldots, t_{N}, \omega\right)-F\left(s_{1}, \ldots, s_{N}, \omega\right)\right|^{p} d \mu(\omega)\right)^{1 / p}$.

An important property of the Choquet $L^{p}$-modulus of continuity used in approximation is the following one, stated and proved here only for simplicity for two variables.

Theorem 3.1. Let $1 \leq p<+\infty$ and $N \in \mathbb{N}$. If $\mu$ is a submodular capacity, then

$$
\Gamma\left(F: \alpha_{1} \cdot \gamma_{1}, \ldots, \alpha_{N} \cdot \gamma_{N}\right)_{p} \leq\left(1+\alpha_{1}+\ldots+\alpha_{N}\right) \Gamma\left(F: \gamma_{1}, \ldots, \gamma_{N}\right)_{p}
$$

for all $\alpha_{i}, \gamma_{i} \geq 0, i=1, \ldots, N$.

Proof. For simplicity, we give the proof only for $N=2$, but the proof in the general case for $N$ is similar. We start with the inequality

$$
\Gamma\left(F: \delta_{1}+\delta_{2}, \eta_{1}+\eta_{2}\right)_{p} \leq \Gamma\left(F: \delta_{1}, \eta_{1}\right)_{p}+\Gamma\left(F: \delta_{2}, \eta_{2}\right)_{p} .
$$

Indeed, let $t_{1}, r_{1}, s_{1}$ with $\left|t_{1}-s_{1}\right| \leq \delta_{1}+\delta_{2},\left|t_{1}-r_{1}\right| \leq \delta_{1},\left|r_{1}-s_{1}\right| \leq \delta_{2}$ and $t_{2}, r_{2}, s_{2}$ with $\left|t_{2}-s_{2}\right| \leq \eta_{1}+\eta_{2},\left|t_{2}-r_{2}\right| \leq \eta_{1},\left|r_{2}-s_{2}\right| \leq \eta_{2}$.

Since $\mu$ is submodular, the Minkowski inequality is valid in the vector space $\mathcal{L}(\Omega, \mathcal{A}, \mu)$, see Theorem 2, p. 5 in [4], or Proposition 9.4, p. 109-110 in [5]. Therefore

$$
\begin{aligned}
\left((C) \int_{\Omega} \mid F\left(t_{1}, t_{2}, \omega\right)\right. & \left.-\left.F\left(s_{1}, s_{2}, \omega\right)\right|^{p} d \mu(\omega)\right)^{1 / p} \\
\leq & \left((C) \int_{\Omega}\left|F\left(t_{1}, t_{2}, \omega\right)-F\left(r_{1}, r_{2}, \omega\right)\right|^{p} d \mu(\omega)\right)^{1 / p} \\
& \quad+\left((C) \int_{\Omega}\left|F\left(r_{1}, r_{2}, \omega\right)-F\left(s_{1}, s_{2}, \omega\right)\right|^{p} d \mu(\omega)\right)^{1 / p} .
\end{aligned}
$$

Passing now to the corresponding suprema, first in the right-hand side and then in the left-hand side, we are led to (3.4). As a consequence

$$
\Gamma(F: n \delta, m \eta)_{p} \leq \max \{n, m\} \Gamma(F: \delta, \eta)_{p}
$$

and taking into account that $\alpha<[\alpha]+1, \beta<[\beta]+1$ and

$$
\max \{[\alpha]+1,[\beta]+1\}=\max \{[\alpha],[\beta]\}+1<\alpha+\beta+1,
$$

one easily obtain the inequality in the statement of Theorem 3.1. 


\section{ApPROXIMATION BY STOCHASTIC BERNSTEIN POLYNOMIALS IN CHOQUET $p$-MEAN}

The approximation of random functions defined on a compact $N$-dimensional interval in $\mathbb{R}^{N}$ (that is, on a product of $N$ compact intervals of $\mathbb{R}$ ) can be easily reduced (via an affine transformation) to the particular case where the domain is the $N$-dimensional unit cube $[0,1]^{N}$. In this context it is important to study the approximation of random functions $F:[0,1]^{N} \rightarrow L^{0}(\Omega, \mathcal{A}, \mu)$ via the associated stochastic Bernstein polynomials,

$$
B_{n_{1}, \ldots, n_{N}}(F)\left(x_{1}, \ldots, x_{N}, \omega\right)=\sum_{k_{1}=0}^{n_{1}} \ldots \sum_{k_{N}=0}^{n_{N}} p_{k_{1}, n_{1}}\left(x_{1}\right) \cdots p_{k_{N}, n_{N}}\left(x_{N}\right) \cdot F\left(\frac{k_{1}}{n_{1}}, \ldots, \frac{k_{N}}{n_{N}}, \omega\right)
$$

where $p_{k_{j}, n_{j}}\left(x_{j}\right)=\left(\begin{array}{c}n_{j} \\ k_{j}\end{array}\right) x_{j}^{k_{j}}\left(1-x_{j}\right)^{n_{j}-k_{j}}, k_{j} \in\left\{0, \ldots, n_{j}\right\}, n_{j} \in \mathbb{N}$ and $x_{j} \in[0,1]$ for $j=1, \ldots N$.

Recall that the classical Bernstein polynomials attached to a function $f:[0,1] \rightarrow \mathbb{R}$ are defined by the formula

$$
B_{n}(f)(x)=\sum_{k=0}^{n} f\left(\frac{k}{n}\right) p_{k, n}(x), \quad x \in[0,1], n \in \mathbb{N},
$$

and their main feature is the estimate

$$
\sup _{x}\left\|B_{n}(f)(x)-f(x)\right\| \leq c \cdot \omega_{1}\left(f ; \frac{1}{\sqrt{n}}\right),
$$

where $c=\frac{4306+837 \sqrt{6}}{5832}=1,089 \ldots$ is the optimal Sikkema constant and

$$
\omega_{1}(f ; \delta)=\sup \{|f(x)-f(y)|: x, y \in[0,1],|x-y| \leq \delta\}
$$

is the usual modulus of continuity, see [24].

The approximation of random functions by stochastic Bernstein polynomials will be discussed in the context of submodular capacity spaces $(\Omega, \mathcal{A}, \mu)$. We consider here the case of approximation in the Choquet $p$-mean, $p \in[1, \infty)$.

Theorem 4.2. Suppose that $(\Omega, \mathcal{A}, \mu)$ is a submodular capacity space and

$$
F:[0,1]^{N} \rightarrow L^{p}(\Omega, \mathcal{A}, \mu)
$$

is a random function. Then for all $x_{1}, x_{2}, \ldots, x_{N} \in[0,1]$ and $n_{1}, n_{2}, \ldots, n_{N} \in \mathbb{N}$, the following quantitative estimate holds

$$
\begin{aligned}
{\left[(C) \int_{\Omega}\left|F\left(x_{1}, x_{2}, \ldots x_{N}, \omega\right)-B_{n_{1}, n_{2}}(F)\left(x_{1}, x_{2}, \ldots, x_{N}, \omega\right)\right|^{p} \mathrm{~d} \mu\right]^{1 / p} } & \\
& \leq\left[C_{p}\right]^{1 / p} \cdot \Gamma\left(F ; \frac{1}{\sqrt{n_{1}}}, \frac{1}{\sqrt{n_{2}}}, \ldots, \frac{1}{\sqrt{n_{N}}}\right)_{p},
\end{aligned}
$$

where $C_{p}$ is independent of $n_{1}, n_{2}, \ldots, n_{N}$ and $x_{1}, x_{2}, \ldots, x_{N}$.

If $F$ is continuous in the Choquet p-mean at each $\mathbf{x} \in[0,1]^{N}$, then the sequence of random Bernstein polynomials $\left(B_{n_{1}, \ldots, n_{N}}(F)\right)_{n_{1}, \ldots, n_{N}}$ converges uniformly to $F$ in the Choquet $p$-mean as $\min \left\{n_{1}, \ldots, n_{N}\right\} \rightarrow \infty$.

Proof. For simplicity, we will give all the details of the proof in the case $N=2$ (the general case being similar). Taking into account the identity

$$
\sum_{k_{1}=0}^{n_{1}} \sum_{k_{2}=0}^{n_{2}} p_{k_{1}, n_{1}}\left(x_{1}\right) \cdot p_{k_{2}, n_{2}}\left(x_{2}\right)=1
$$


and the convexity of the function $x^{p}$ for $p \geq 1$, we infer from Jensen's inequality that

$$
\begin{aligned}
& \left|F\left(x_{1}, x_{2}, \omega\right)-B_{n_{1}, n_{2}}(F)\left(x_{1}, x_{2}, \omega\right)\right|^{p} \\
& \leq\left[\sum_{k_{1}=0}^{n_{1}} \sum_{k_{2}=0}^{n_{2}} p_{k_{1}, n_{1}}\left(x_{1}\right) \cdot p_{k_{2}, n_{2}}\left(x_{2}\right) \mid F\left(x_{1}, x_{2}, \omega\right)-F\left(k_{1} / n_{1}, k_{2} / n_{2}, \omega\right)\right]^{p} \\
& \quad \leq \sum_{k_{1}=0}^{n_{1}} \sum_{k_{2}=0}^{n_{2}} p_{k_{1}, n_{1}}\left(x_{1}\right) \cdot p_{k_{2}, n_{2}}\left(x_{2}\right)\left|F\left(x_{1}, x_{2}, \omega\right)-F\left(k_{1} / n_{1}, k_{2} / n_{2}, \omega\right)\right|^{p} .
\end{aligned}
$$

Integrating side by side and using Remark 2.1, (a) and (b) we arrive at the estimate

$$
\text { (C) } \int_{\Omega}\left|F\left(x_{1}, x_{2}, \omega\right)-B_{n_{1}, n_{2}}(F)\left(x_{1}, x_{2}, \omega\right)\right|^{p} \mathrm{~d} \mu
$$

$$
\leq \sum_{k_{1}=0}^{n_{1}} \sum_{k_{2}=0}^{n_{2}} p_{k_{1}, n_{1}}\left(x_{1}\right) p_{k_{2}, n_{2}}\left(x_{2}\right)(C) \int_{\Omega}\left|F\left(x_{1}, x_{2}, \omega\right)-F\left(k_{1} / n_{1}, k_{2} / n_{2}, \omega\right)\right|^{p} \mathrm{~d} \mu .
$$

Using the inequality (4.6) and then Theorem 3.1, we get

$$
\begin{gathered}
(C) \int_{\Omega}\left|F\left(x_{1}, x_{2}, \omega\right)-B_{n_{1}, n_{2}}(F)\left(x_{1}, x_{2}, \omega\right)\right|^{p} \mathrm{~d} \mu \leq \sum_{k_{1}=0}^{n_{1}} \sum_{k_{2}=0}^{n_{2}} p_{k_{1}, n_{1}}\left(x_{1}\right) p_{k_{2}, n_{2}}\left(x_{2}\right) \\
\qquad\left[\Gamma\left(F ; \frac{1}{\sqrt{n_{1}}} \cdot\left(\sqrt{n_{1}}\left|x_{1}-k_{1} / n_{1}\right|\right), \frac{1}{\sqrt{n_{2}}} \cdot\left(\sqrt{n_{2}}\left|x_{2}-k_{2} / n_{2}\right|\right)\right)_{p}\right]^{p} \\
\left.\leq\left[\Gamma ; \frac{1}{\sqrt{n_{1}}}, \frac{1}{\sqrt{n_{2}}}\right)_{p}^{p}\right]^{p} \cdot \sum_{k_{1}=0}^{n_{1}} \sum_{k_{2}=0}^{n_{2}} p_{k_{1}, n_{1}}\left(x_{1}\right) p_{k_{2}, n_{2}}\left(x_{2}\right)\left(1+\sqrt{n_{1}}\left|x_{1}-k_{1} / n_{1}\right|+\sqrt{n_{2}}\left|x_{2}-k_{2} / n_{2}\right|\right)^{p} .
\end{gathered}
$$

But by the general estimates of the moments of Bernstein polynomials

$$
\sum_{k=0}^{n} p_{k, n}(x)[\sqrt{n}|x-k / n|]^{j} \leq 2 G(1+j / 2), j=0,1, \ldots, p,
$$

where with $G$ we have denoted the Gamma function (see Theorem 1 in J. A. Adell, J. Bustamente and J. M. Quesada [1]), it is immediate that

$$
\sum_{k_{1}=0}^{n_{1}} \sum_{k_{2}=0}^{n_{2}} p_{k_{1}, n_{1}}\left(x_{1}\right) p_{k_{2}, n_{2}}\left(x_{2}\right)\left(1+\sqrt{n_{1}}\left|x_{1}-k_{1} / n_{1}\right|+\sqrt{n_{2}}\left|x_{2}-k_{2} / n_{2}\right|\right)^{p} \leq C_{p},
$$

where $C_{p}$ is independent of $n_{1}, n_{2}$ and $x_{1}, x_{2} \in[0,1]$. Concluding, we obtain

$$
\left[(C) \int_{\Omega}\left|F\left(x_{1}, x_{2}, \omega\right)-B_{n_{1}, n_{2}}(F)\left(x_{1}, x_{2}, \omega\right)\right|^{p} \mathrm{~d} \mu\right]^{1 / p} \leq\left[C_{p}\right]^{1 / p} \cdot \Gamma\left(F ; \frac{1}{\sqrt{n_{1}}}, \frac{1}{\sqrt{n_{2}}}\right)_{p} .
$$

On the other hand, we observe that the continuity of $F$ in the Choquet $p$-mean at each $x$ in the compact $[0,1]^{N}$, easily implies its uniform continuity on $[0,1]^{N}$, which by the definition of the multivariate Choquet $L^{p}$-modulus of continuity of $F$, immediately implies that $\lim _{\delta_{1}, \ldots, \delta_{N} \rightarrow 0} \Gamma\left(F ; \delta_{1}, \ldots, \delta_{N}\right)_{p}=0$. This implies the second part of the theorem too. 
Remark 4.2. The particular case of Theorem 4.2 , when $\mu$ is a $\sigma$-additive measure, $N=1$ and $p=2$, was previously proved by Ignatov, Mills and Tzankova [19] and Kamolov [20]. Also, the second part of Theorem 4.2 , for $\mu$ a $\sigma$-additive measure, $N=1$ and $p=1$ was first noticed by Cenuşă and Săcuiu [3].

\section{QUANTITATIVE ESTIMATES FOR CONVERGENCE IN CAPACITY}

This section is devoted to the proof of several quantitative estimates for the approximation in capacity by univariate stochastic Bernstein-type polynomials. Our results were inspired by the recent papers of Adell and Cárdenas-Morales [2], Sun and Wu [25], Wu, Sun and Ma [26] and $\mathrm{Wu}$ and Zhou [27], who considered only the framework of probability measures and of deterministic functions.

In the definition of the classical univariate Bernstein polynomials, the function $f$ : $[0,1] \rightarrow \mathbb{R}$ is evaluated at the set of equally spaced nodes $k / n, k=0,1, \ldots, n$. However, in real problems, data at equally spaced nodes are sometimes contaminated by random errors due to a variety of factors.

Thus, in this section, we consider the approximation in capacity of a random function $f(x, \omega)$ by stochastic Bernstein polynomials

$$
B_{n}(f, Y)(x, \omega)=\sum_{k=0}^{n} f\left(Y_{n, k}(\omega), \omega\right) p_{k, n}(x),
$$

where $Y=\left\{Y_{n, k}: n \in \mathbb{N}, k=0, \ldots, n\right\}$ is a triangular array of random variables $Y_{n, k}$ : $\Omega \rightarrow \mathbb{R}$, such that

$$
0 \leq Y_{n, 0} \leq Y_{n, 1} \leq \ldots . \leq Y_{n, n} .
$$

We shall need the following two quantities associated to a random function $f$ :

$$
\omega_{1, x}(f ; \delta)(\omega)=\sup \{|f(x, \omega)-f(y, \omega)|: x, y \in[0,1],|x-y| \leq \delta\}
$$

and

$$
K(f ; \delta)=\sup \left\{\omega_{1, x}(f ; \delta)(\omega): \omega \in \Omega\right\}, \quad \delta \geq 0 .
$$

It is immediate that $K(f ; \delta)$ is nondecreasing and subadditive as function of $\delta \geq 0$. Also, it is easy to see that if $f(x, \omega)$ is continuous at each $x \in[0,1]$, uniformly with respect to $\omega$, then $\lim _{\delta \rightarrow 0} K(f ; \delta)=0$, so in this case $K(f ; \cdot)$ is a modulus of continuity.

We put

$$
M_{n}(\omega)=\max \left\{\left|Y_{n, k}(\omega)-\frac{k}{n}\right|: 0 \leq k \leq n\right\}, \quad n \in \mathbb{N}, \omega \in \Omega .
$$

Theorem 5.3. Let $f:[0,1] \times \Omega \rightarrow \mathbb{R}$ be continuous at each $x \in[0,1]$, uniformly with respect to $\omega \in \Omega, \mathcal{C}$ a $\sigma$-algebra of subsets of $\Omega$ and $\mu: \mathcal{C} \rightarrow[0,1]$ a capacity. If

$$
\lim _{n \rightarrow \infty} \mu\left(\left\{\omega \in \Omega: M_{n}(\omega)>\varepsilon\right\}\right)=0,
$$

for every $\varepsilon>0$, then $B_{n}(f, Y)(x, \omega)$ converges to $f(x, \omega)$ in capacity, uniformly with respect to $x \in[0,1]$.

In addition, for every $0<\delta<1$ and $n \geq 1 / \delta^{2}$, we have

$$
\begin{aligned}
& \mu\left(\left\{\omega \in \Omega: \sup _{x}\left\|B_{n}(f, Y)(x, \omega)-f(x, \omega)\right\|>(1+c) K(f, \delta)\right\}\right) \\
& \leq \mu\left(\left\{\omega \in \Omega: M_{n}(\omega)>\delta\right\}\right),
\end{aligned}
$$

where $c$ is the Sikkema constant. 
Proof. First, let $B_{n}(f)(x, \omega)=\sum_{k=0}^{n} f\left(\frac{k}{n}, \omega\right) p_{k, n}(x)$. If we fix $\omega \in \Omega$, then by repeating the argument used by Sikkema [24] for the formula (4.5), we immediately get

$$
\begin{aligned}
& \sup _{x}\left\|B_{n}(f)(x, \omega)-f(x, \omega)\right\| \\
\leq & c \cdot \sup \left\{|f(x, \omega)-f(y, \omega)|: x, y \in[0,1],|x-y| \leq \frac{1}{\sqrt{n}}\right\} \leq c \cdot K\left(f ; \frac{1}{\sqrt{n}}\right), \text { for } \omega \in \Omega, n \in \mathbb{N} .
\end{aligned}
$$

For $x \in[0,1]$ and $\omega_{0} \in \Omega$, by the triangular inequality it follows that

$$
\begin{array}{r}
\left|B_{n}(f, Y)\left(x, \omega_{0}\right)-f\left(x, \omega_{0}\right)\right|=\left|\sum_{k=0}^{n}\left[f\left(Y_{n, k}\left(\omega_{0}\right), \omega_{0}\right)-f\left(x, \omega_{0}\right)\right] p_{k, n}(x)\right| \\
\leq \sup _{x}\left\|B_{n}(f)\left(x, \omega_{0}\right)-f\left(x, \omega_{0}\right)\right\|+\sum_{K=0}^{n} \omega_{1, x}\left(f ;\left|Y_{n, k}\left(\omega_{0}\right)-k / n\right|\right)\left(\omega_{0}\right) \cdot p_{k, n}(x) \\
\leq c \cdot K\left(f ; \frac{1}{\sqrt{n}}\right)+\omega_{1, x}\left(f ; M_{n}\left(\omega_{0}\right)\right)\left(\omega_{0}\right) \leq c \cdot K\left(f ; \frac{1}{\sqrt{n}}\right)+K\left(f ; M_{n}\left(\omega_{0}\right)\right),
\end{array}
$$

since

$$
\begin{gathered}
\omega_{1, x}\left(f ; M_{n}\left(\omega_{0}\right)\right)\left(\omega_{0}\right)=\sup \left\{\left|f\left(x, \omega_{0}\right)-f\left(y, \omega_{0}\right)\right|: x, y \in[0,1],|x-y| \leq M_{n}\left(\omega_{0}\right)\right\} \\
\leq \sup \left\{\sup \{|f(x, \omega)-f(y, \omega)|: \omega \in \Omega\}: x . y \in[0,1],|x-y| \leq M_{n}\left(\omega_{0}\right)\right\} \\
=\sup \left\{\sup \left\{|f(x, \omega)-f(y, \omega)|: x, y \in[0,1],|x-y| \leq M_{n}\left(\omega_{0}\right)\right\}: \omega \in \Omega\right\}=K\left(f ; M_{n}\left(\omega_{0}\right)\right) .
\end{gathered}
$$

For $\varepsilon \in(0, K(f ; 1))$ and $K\left(f ; \frac{1}{\sqrt{n}}\right) \leq \varepsilon$, from the previous estimate, by the monotonicity of $\mu$ and by Lemma 1 in Adell and Cárdenas-Morales [2], we infer that

$$
\begin{gathered}
\mu\left(\left\{\omega_{0} \in \Omega: \sup _{x}\left\|B_{n}(f, Y)\left(x, \omega_{0}\right)-f\left(x, \omega_{0}\right)\right\|>(1+c) \varepsilon\right\}\right) \\
\leq \mu\left(\left\{\omega_{0} \in \Omega ; K\left(f ; M_{n}\left(\omega_{0}\right)\right)>\varepsilon\right\}\right)=\mu\left(\left\{\omega_{0} \in \Omega: M_{n}\left(\omega_{0}\right)>\tilde{K}(f ; \varepsilon)\right\},\right.
\end{gathered}
$$

where $\tilde{K}$ is the right-continuous inverse of the modulus of continuity $K$, given by formula (5.8) and satisfying

$$
\delta \leq \tilde{K}(f ; K(f ; \delta)), \quad \text { for all } 0 \leq \delta \leq 1 .
$$

Indeed, the equality in (5.11) follows immediately from the nondecreasing monotonicity of $K(f ; \cdot)$ and $\tilde{K}(f ; \cdot)$ and applying $\tilde{K}(f ; \cdot)$ to $K\left(f ; M_{n}\left(\omega_{0}\right)\right)>\varepsilon$ and $K(f ; \cdot)$ to $M_{n}\left(\omega_{0}\right)>$ $\tilde{K}(f ; \varepsilon)$.

Choosing now $\varepsilon=K(f ; \delta)$ with $\delta \geq \frac{1}{\sqrt{n}}$ in (5.11), taking into account (5.12) and from $K\left(f ; \frac{1}{\sqrt{n}}\right) \leq \varepsilon$, it follows the estimate in the statement.

Remark 5.3. If $\mu$ is a probability measure and $f$ is deterministic, then $K(f, \delta)$ becomes the usual modulus of continuity and by Theorem 5.3 we obtain Theorem 1 in Adell and Cárdenas-Morales [2].

In the next lemma we shall need the triangular array $Y$ obtained as follows. For each $n \in \mathbb{N}$, let $\left(V_{j}\right)_{j=1}^{n+1}$ be a finite sequence of independent identically distributed random variables having the uniform distribution on [0,1]. Let $V_{n+1: 1} \leq \cdots \leq V_{n+1: n+1}$ be the order statistics obtained by arranging $\left(V_{j}\right)_{j=1}^{n+1}$ in increasing order and put

$$
Y=\left\{Y_{n, k}=V_{n+1: k+1}, n \in \mathbb{N}, k=0,1, \ldots, n\right\} .
$$


Lemma 5.1. Suppose that $\mu: \mathcal{C} \rightarrow[0,1]$ is a distorted probability of the form $\mu=u \circ P$, where $P$ is a probability measure and $u:[0,1] \rightarrow \mathbb{R}$ is a strictly increasing and concave function such that $u(0)=0$ and $u(1)=1$. If $0<u^{\prime}(0)<\infty$, then for every $\varepsilon>0, n \in \mathbb{N}$ and $0<r<1$ we have

$$
\mu\left(\left\{\omega \in \Omega: M_{n}(\omega)>\varepsilon\right\}\right) \leq u^{\prime}(0) \cdot \frac{n+1}{\sqrt{1-r}} \cdot \exp \left(-\frac{3 r}{2} n \varepsilon^{2}\right),
$$

where exp denotes the exponential function and $M_{n}(\omega)$ is defined by

$$
M_{n}(\omega)=\max \left\{\left|Y_{n, k}(\omega)-\frac{k}{n}\right|: 0 \leq k \leq n\right\} \quad \text { for } n \in \mathbb{N}, \omega \in \Omega .
$$

Proof. Clearly, $x \leq u(x) \leq u^{\prime}(0) x$ for all $x \in[0,1]$, which for $x=\mu(A)$ gives us $\mu(A) \leq$ $u^{\prime}(0) P(A)$ for all $A \in \mathcal{C}$. Combining this fact with the estimate

$$
P\left(M_{n}>\varepsilon\right) \leq \frac{n+1}{\sqrt{1-r}} \exp \left(-\frac{3 r}{2} n \varepsilon^{2}\right),
$$

in Adell and Cárdenas-Morales [2], Lemma 2, p. 7, the Lemma 5.1 is proved.

Theorem 5.4. Let $(\tau(n))_{n}$ satisfying the conditions

$$
\lim _{n \rightarrow \infty} \tau(n)=\infty, \lim _{n \rightarrow \infty} \frac{\tau(n)}{n}=0 \text { and } \tau(n) \geq 1 \text { for } n \in \mathbb{N},
$$

let $Y$ be the triangular array (5.13) and let $\mu$ the distorted probability defined as in Lemma 5.1. Then, for any random function $f(x, \omega)$ continuous at each $x \in[0,1]$ uniformly with respect to $\omega$, for any $r \in(0,1)$ and any $n \in \mathbb{N}$, we have

$$
\begin{aligned}
& \mu\left(\left\{\omega \in \Omega: \sup _{x}\left\|B_{n}(f, Y)(x, \omega)-f(x, \omega)\right\|>(1+c) K\left(f ; \sqrt{\frac{\tau(n)}{n}}\right)\right\}\right) \\
& \leq u^{\prime}(0) \cdot \frac{n+1}{\sqrt{1-r}} \exp \left(-\frac{3 r}{2} \tau(n)\right) .
\end{aligned}
$$

Here $c$ is the Sikkema's constant.

Proof. Choosing $\delta=\sqrt{\frac{\tau(n)}{n}}$ in Theorem 5.3, we have $n \geq \frac{1}{\delta^{2}}$ since $\tau(n) \geq 1$. The proof ends by applying Lemma 5.1 .

There are many examples of distorted probabilities $\mu=u \circ P$ satisfying the hypothesis of Theorem 5.4. One can choose $u(t)=\frac{2 t}{t+1}, u(t)=\left(1-e^{-t}\right) /\left(1-e^{-1}\right), u(t)=\ln (1+$ $t) / \ln (2), u(t)=\sin (\pi t / 2)$, or $u(t)=\frac{4}{\pi} \cdot \arctan (t)$, for $t \in[0,1]$.

If $\mu$ is a probability measure (that is, when $u(t)=t$ ) and $f$ is a deterministic function, then Theorem 5.4 reduces to Corollary 1 in Adell and Cárdenas-Morales [2].

\section{REFERENCES}

[1] Adell, J. A., Bustamante, J. and Quesada, J. M., Estimates for the moments of Bernstein polynomials, J. Math. Anal. Appl, 432 (2015), 114-128

[2] Adell, J. A. and Cárdenas-Morales, D., Stochastic Bernstein polynomials: uniform convergence in probability with rates, Adv. Comput. Math., 46 (2020), Art. 16, 10 pp.

[3] Cenuşă, Gh. and Săcuiu, I., On some stochastic approximations for random functions, Rend. Mat., Serie VI, 12 (1979), 143-156

[4] Cerdà, J., Martín, J. and Silvestre P., Capacitary function spaces, Collect. Math., 62 (2011), 95-118

[5] Denneberg, D., Non-Additive Measure and Integral, Kluwer Academic Publisher, Dordrecht, 1994

[6] Föllmer, H. and Schied, A., Stochastic Finance, Fourth revised and extended edition, De Gruyter, 2016

[7] Gal, S. G., Jackson type estimates in the approximation of random functions by random polynomials, Rend. Mat. Appl., (7) 14 (1994), No. 4, 543-556 
[8] Gal, S. G., Approximation theory in random setting, Chapter 12 in Handbook of analytic-computational methods in applied mathematics, pp. 571-616. Chapman and Hall/CRC, Boca Raton, FL, 2000

[9] Gal, S. G., Approximation by nonlinear Choquet integral operators, Annali di Mat. Pura Appl., 195 (2016), No. 3, 881-896

[10] Gal, S. G. and Iancu, I. T., Quantitative approximation by nonlinear convolution operators of Landau-Choquet type, Carpath. J. Math., 36 (2020), 415-422

[11] Gal, S. G. and Niculescu, C. P., Kantorovich's mass transport problem for capacities, Proc. Rom. Acad. Ser. A, Math. Phys. Tech. Sci. Inf. Sci., 20 (2019), No. 4, 337-345

[12] Gal, S. G. and Niculescu, C. P., A nonlinear version of Korovkin's theorem, Mediterr. J. Math., 17 (2020), Issue 5 , Article No. 145

[13] Gal, S. G. and Niculescu, C. P., A note on Choquet type operators, Aequat. Math., DOI:10.10007/s00010-021-0803-z

[14] Gal, S .G. and Niculescu, C. P., Choquet operators associated to vector capacities, J. Math. Anal. Appl., 500 (2021), Issue 2, Article No. 125153

[15] Gal, S. G. and Opris, B. D., Uniform and pointwise convergence of Bernstein-Durrmeyer operators with respect to monotone and submodular set functions, J. Math. Anal. Appl., 424 (2015), 1374-1379

[16] Gal, S. G. and Trifa, S., Quantitative estimates in uniform and pointwise approximation by Bernstein-DurrmeyerChoquet operators, Carpath. J. Math., 33 (2017), 49-58

[17] Gal, S. G. and Villena, A. R., Random condensation of singularities and applications, Random Oper. Stochastic Equations 5 (1997), No. 3, 263-268

[18] Grabisch, M., Set Functions, Games and Capacities in Decision Making, Theory and Decision Library C (Game Theory, Social Choice, Decision Theory, and Optimization), 46, Springer, 2016

[19] Ignatov, Z. G., Mills, T. M. and Tzankova, I. P., On the rate of approximation of random functions, Serdica, Bulgaricae mathematicae publicationes, 18 (1992), 240-247

[20] Kamolov, A. I., On exact estimates of approximation of random processes (in Russian), Dokl. Akad. Nauk. UzSSR, 11 (1986), 4-6

[21] Lorentz, G. G., Bernstein Polynomials, Second edition. Chelsea Publishing Company, New York, 1986

[22] Onicescu, O. and Istrăţescu, V. I., Approximation theorems for random functions, Rend. Mat., Serie VI, 8 (1975), 65-81

[23] Onicescu, O. and Istrățescu, V. I., Approximation theorems for random functions, II, Rend. Mat. Serie VI, 11 (1978), No. 4, 585-589

[24] Sikkema, P. C., Der Wert einiger Konstanten in der Theorie der Approximation mit Bernstein- Polynomen, Numer. Math, 3 (1961), 107-116

[25] Sun, X. and Wu, Z., Chebyshev type inequality for stochastic Bernstein polynomials, Proc. Amer. Math. Soc., 147 (2019), No. 2, 671-679

[26] Wu, Z., Sun, X. and Ma, L., Sampling scattered data with Bernstein polynomials: stochastic and deterministic error estimates, Adv. Comput. Math., 38 (2020), 187-205

[27] Wu, Z. and Zhou, X., Polynomial convergence order of stochastic Bernstein approximation, Adv. Comput. Math. 46 (2020), Art. 8, 14 pp.

Department of MATHEMATICS ANd COMPUter SCIENCE

UNIVERSITY OF ORADEA

UNIVERSITĂŢII 1, 410087, ORADEA, ROMANIA

Email address: gal so@uoradea.ro

Department of MATHEMATiCs

UNIVERSITY OF CRAIOVA

CRAIOVA, 200585, ROMANIA

Email address: constantin.p.niculesculgmail.com 